\title{
Influence of orthodontic brackets and permanent retainers on the diagnostic image quality of MRI scans: A preliminary study
}

\author{
Praveen Kumar Neela ${ }^{1, A, B, D-F}$, Venkat Kishan Tatikonda ${ }^{2, A-F}$, Mohammed Wahajuddin Syed ${ }^{1, A, B, D, F}$, Pavan Kumar Mamillapalli ${ }^{1, A-D, F}$, \\ Vasu Murthy Sesham ${ }^{1, A, C, D, F}$, Sreekanth Keesara ${ }^{1, A, B, D, F}$ \\ ${ }^{1}$ Department of Orthodontics, Kamineni Institute of Dental Sciences, Sreepuram, Narketpally, India \\ ${ }^{2}$ Department of Radio-Diagnosis, Kamineni Institute of Medical Sciences, Sreepuram, Narketpally, India \\ A - research concept and design; $B$ - collection and/or assembly of data; $C$ - data analysis and interpretation; \\ $D$ - writing the article; $E$ - critical revision of the article; $F$ - final approval of the article
}

\section{Address for correspondence \\ Praveen Kumar Neela \\ E-mail: praveenneela@yahoo.com}

Funding sources

None declared

Conflict of interest

None declared

Acknowledgements

We are grateful to the subjects who participated in the study. We would also like to thank the 6 radiologists for devoting their valuable time to analyzing the MRI scans.

Received on September 28, 2020

Reviewed on January 11, 2021

Accepted on January 13, 2021

Published online on December 14, 2021

Cite as

Neela PK, Tatikonda VK, Syed MW, Mamillapalli PK, Sesham VM, Keesara S. Influence of orthodontic brackets and permanent retainers on the diagnostic image quality of MRI scans: A preliminary study. Dent Med Probl. 2021;58(4):499-508. doi:10.17219/dmp/132390

DOI

$10.17219 / \mathrm{dmp} / 132390$

Copyright

○) 2021 by Wroclaw Medical University

This is an article distributed under the terms of the

Creative Commons Attribution 3.0 Unported License (CC BY 3.0)

(https://creativecommons.org/licenses/by/3.0/).

\begin{abstract}
Background. Orthodontic treatment with fixed mechanotherapy using appliances and permanent retainers bonded after treatment is a routine procedure performed in clinical dentistry. Patients with braces or retainers sometimes need to undergo magnetic resonance imaging (MRI) for various reasons. Radiologists do not know the exact impact of the materials used in orthodontics on the diagnostic image quality of MRI scans.
\end{abstract}

Objectives. The aim of the study was to evaluate the influence of different types of orthodontic brackets and permanent retainers on the diagnostic image quality of MRI scans.

Material and methods. Twenty patients with bonded brackets (stainless steel buccal/labial, stainless steel lingual, ceramic self-ligating with metal slots, ceramic, and polycarbonate) and 18 patients with bonded fixed retainers (titanium, fiber-reinforced composite, multi-stranded stainless steel, and different combinations of permanent retainers) participated in the study. The same adhesive was used for bonding. Cranial MRI scans of 6 regions were acquired for each subject, using a 1.5T MAGNETOM machine. Six radiologists evaluated the images and provided scores based on the modified receiver operating characteristic (ROC) analysis of distortion. The paired Wilcoxon signed-rank test was used to assess differences between the materials and the anatomic sites with regard to the distortion rating scale. Cohen's kappa coefficient ( $k$ ) was applied to establish the interrater reliability.

Results. A statistically significant difference was found between stainless steel brackets (both buccal/ labial and lingual) and all other experimental materials in terms of mean distortion scores ( $p=0.020$ or $p=0.024$ ). The interrater reliability proved to be high.

Conclusions. Stainless steel buccal/labial and lingual brackets caused maximum distortion of the images, which became non-diagnostic; hence, such brackets should be removed before MRI. Ceramic and polycarbonate brackets as well as fiber-reinforced composite retainers did not distort the images; thus, they need not be removed. Ceramic self-ligating brackets with metal slots, titanium retainers, multi-stranded stainless steel retainers, and combinations of fixed retainers caused minimal distortion; however, the images were still diagnostic. Hence, patients using these materials may not need to have them removed before MRI.

Keywords: magnetic resonance imaging, orthodontic brackets, bonded retainers, MRI distortion 


\section{Introduction}

Magnetic resonance imaging (MRI) is one of the most powerful diagnostic tools in radiology. It is characterized by high sensitivity and specificity. Besides, it is a noninvasive procedure. The system does not use ionizing radiation, and for the majority of people there is little risk associated with the application of the magnetic field. This radiological modality is indispensable when investigating soft tissue tumors, including those of the head and neck, ${ }^{1}$ temporomandibular joint (TMJ) pathology, ${ }^{2}$ cardiovascular pathology, ${ }^{3}$ seizures, ${ }^{4}$ and cerebral palsy. ${ }^{5}$ The number of MRI scans performed per year is steadily increasing.

Fixed orthodontic treatment is a standard and increasingly prescribed practice for both children and adults. It involves bonding orthodontic brackets in the patient's mouth for the duration of treatment, which generally lasts 24-30 months. ${ }^{6}$ Orthodontic treatment is routinely followed by long-term retention to maintain the treatment result with the use of a metal or non-metal wire that is fixed to the lingual surface of the lower anterior teeth and remains in situ for several years. ${ }^{7}$

Patients with fixed multibracket orthodontic appliances sometimes require the MRI examination of the head and neck region for various reasons. These may include diagnosing epilepsy, recurrent headaches, trauma, and the pathology of the salivary glands, tongue and TMJ. While it is known that fixed multibracket orthodontic appliances cause MRI artifacts, the extent and severity of image loss is not clearly described in the literature. The heating of the appliance which occurs during MRI, although negligible, is the reason why it may be necessary to remove the orthodontic appliances or archwires to prevent artifacts. ${ }^{8,9}$ This increases the financial and biological burdens, since the removal and later reinstallation of fixed orthodontic appliances are costly, labor-intensive ${ }^{10}$ and time-consuming, and require the remodeling of the bone once again. However, the removal of archwires is easy and less time-consuming. ${ }^{11}$

A few studies have reported that the artifacts caused by stainless steel brackets and wires do result in some distortion of the MR images of the brain; however, these images remained diagnostic. ${ }^{12,13}$ On the other hand, other studies have reported that orthodontic appliances can render TMJ and brain MR images non-diagnostic. ${ }^{14}$ There is a lack of consensus regarding the best practice protocols to employ for patients who need MRI during the period of orthodontic treatment or retention with fixed appliances. There are, however, guidelines from the Polish Orthodontic Society, the Polish Medical Radiological Society and the Polish Dental Association. ${ }^{15}$ Most of the studies regarding stainless steel brackets were performed on skulls and in volunteers for whom MRI was used without bonding the brackets on the teeth, but instead incorporating them in vacuum-formed sheets, which are then worn by volunteers. Moreover, the effects of some recently introduced brackets, such as ceramic self-ligating brackets with metal slots, ceramic brackets, polycarbonate brackets, and different types of lingual retainers on the diagnostic image quality of MRI scans have not been researched. Although there are guidelines ${ }^{15}$ and literature regarding MRI artifacts that are due to brackets and some wires, this may be the first comprehensive in vivo study to evaluate the diagnostic image quality of MRI scans in patients with different types of orthodontic brackets and fixed retainers. Therefore, this study aimed to evaluate the influence of different types of orthodontic brackets and permanent retainers bonded in orthodontic patients on the diagnostic image quality of MRI scans.

\section{Material and methods}

\section{Ethical statement}

This research was conducted after obtaining approval from the institutional ethics committee at Kamineni Institute of Dental Sciences, Sreepuram, Narketpally, India (KIDS/ IEC/2015/22). The study was carried out following the standards set out in the Declaration of Helsinki for experiments involving humans. Informed consent was obtained from all subjects, who voluntarily participated in the study. They were healthy patients in the age range of 18-25 years.

\section{Patient selection}

Thirty-eight subjects who met the following inclusion criteria were recruited for the study: no metal medical devices, like aneurysmal clips and pacemakers; no metal dental fillings, metal-containing crowns or dental implants; no pregnancy; and no requirement for sedation during MRI. All 38 subjects who volunteered to participate in the study were about to undergo fixed orthodontic treatment or about to start the retention phase. The purpose of the study, including its possible risks, was explained to the participants.

The types of brackets/retainers and the corresponding numbers of patients who underwent MRI were as follows: - stainless steel buccal/labial brackets for all teeth erupted, from anterior ones to second premolars in both the maxillary and mandibular arch, without molar bands and wires (Unitek ${ }^{\mathrm{TM}}$ Gemini; 3M Unitek, Monrovia, USA): 4 patients; - stainless steel lingual brackets for all teeth erupted, from anterior ones to second premolars in both the maxillary and mandibular arch, without molar bands and wires (Invisible 7G; Classic Orthodontics, Stafford, USA): 4 patients;

- ceramic self-ligating brackets with metal slots made of stainless steel for all teeth erupted, from anterior ones to second premolars in both the maxillary and mandibular arch, without molar bands and wires (Unitek Clarity SL; 3M Unitek): 4 patients;

- ceramic brackets for all teeth erupted, from anterior ones to second premolars in both the maxillary and mandibular arch, without molar bands and wires (Inspire ICE; Ormco, Brea, USA): 4 patients; 
- polycarbonate brackets for all teeth erupted, from anterior ones to second premolars in both the maxillary and mandibular arch, without molar bands and wires (Ora-plus; JJ Orthodontics, Munipara, India): 4 patients;

- titanium retainers bonded from right canine to left canine in the mandibular arch only (Ortho-Direct, West Columbia, USA): 4 patients;

- fiber-reinforced composite retainers bonded from right canine to left canine in the mandibular arch only (everStick ${ }^{\circledR}$ ORTHO; GC America, Alsip, USA): 4 patients;

- multi-stranded stainless steel retainers in the maxillary and mandibular arches: 4 patients;

- combinations of these fixed retainers bonded from right canine to left canine in the maxillary and mandibular arches, including:

- a stainless steel retainer in the maxillary arch and a titanium retainer in the mandibular arch: 3 patients,

- a stainless steel retainer in the maxillary arch and a fiberreinforced composite retainer in the mandibular arch: 3 patients.

Thus, a total of 38 subjects (20 with different brackets and 18 with different retainers) participated in the study.

\section{MRI protocol}

A 1.5 Tesla Siemens MAGNETOM MRI machine (Siemens, Erlangen, Germany) with a 12-channel head coil was used.

Six regions of interest (ROI) were analyzed: TMJ; the tongue; the maxilla; the mandible; the maxillary sinus; and the posterior cerebral fossa.

For the posterior cerebral fossa, the MRI brain protocol was used; it is shown in Table 1.

Diffusion-weighted (DW) images were acquired with a single-shot spin-echo echo planar imaging (SE-EPI) sequence (repetition time (TR)/echo time (TE): $5,000 \mathrm{~ms} / 100 \mathrm{~ms}$ ) using 3 values of $0 \mathrm{~s} / \mathrm{mm}^{2}$, $500 \mathrm{~s} / \mathrm{mm}^{2}$ and $1,000 \mathrm{~s} / \mathrm{mm}^{2}$, with a slice thickness of $5 \mathrm{~mm}$, an inter-slice gap of $1.5 \mathrm{~mm}$, a field of view (FoV) of $240 \mathrm{~mm} \times 240 \mathrm{~mm}$, and a matrix of $128 \times 128$. Apparent diffusion coefficient (ADC) maps were generated from the DW image datasets. For susceptibility-weighted imaging (SWI), TR/TE was $6,400 \mathrm{~ms} / 30 \mathrm{~ms}$, with a slice thickness of $5 \mathrm{~mm}$, an inter-slice gap of $1.5 \mathrm{~mm}$, an FoV of $240 \mathrm{~mm} \times 240 \mathrm{~mm}$, and a matrix of $256 \times 256$.
For TMJ, sagittal, axial and coronal proton density (PD)weighted and turbo spin-echo (TSE) transverse relaxation time (T2)-weighted sequences were employed with TR $5,653 \mathrm{~ms}$, TE $13 \mathrm{~ms}$ and $102 \mathrm{~ms}$, a slice thickness of $2 \mathrm{~mm}$, an inter-slice gap of $0.2 \mathrm{~mm}$, an FoV of $160 \mathrm{~mm} \times 160 \mathrm{~mm}$, and a matrix of $256 \times 256$. For the remaining regions, the MRI neck protocol was used. Axial and coronal TSE T2-weighted images were acquired with TR 5,600 ms, TE $114 / 7 \mathrm{~ms}$, a slice thickness of $5 \mathrm{~mm}$, an inter-slice gap of $0.5 \mathrm{~mm}$, an FoV of $240 \mathrm{~mm} \times 240 \mathrm{~mm}$, and a matrix of $320 \times 320$.

No paramagnetic agent was used, as there was no indication for the use of a paramagnetic agent.

Magnetic resonance imaging scans were taken for all 38 subjects to study diagnostic image quality. Since the orthodontic appliances were securely bonded with orthodontic composite, there were no special precautions taken regarding the possible dislodgement and heating of the appliances, as previous studies had found changes in the temperature of the appliances to be clinically insignificant (within $1^{\circ} \mathrm{C}$ ). ${ }^{16}$ The MRI scans for all types of bracket and retainer materials are shown in Fig. 1-10. Non-diagnostic or unclear ROI are marked with arrows. None of the patients reported any discomfort or pain during MRI examination. The MRI scans were analyzed by a panel of 6 qualified and licensed radiologists, who assessed their diagnostic quality. The scans were compared with controls that included images from the archives of the Department of RadioDiagnosis of Kamineni Institute of Medical Sciences. The radiologists ranked the images according to the distortion observed in the abovementioned ROI, using the modified receiver operating characteristic (ROC) analysis of distortion scoring system (Table 2), as described by Elison et al. ${ }^{17}$ In this method of distortion classification, a score of 3 represents the cut-off point for clinical usability. Images with a score of 3 have moderate distortion or artifacts, but they can still be used for diagnosis.

Table 2. Modified receiver operating characteristic (ROC) analysis of distortion scoring system

\begin{tabular}{|c|c|c|}
\hline Score & Image appearance & Inference \\
\hline 1 & no distortion or artifacts & diagnostic \\
2 & minimal distortion or artifacts & diagnostic \\
3 & moderate distortion or artifacts & moderately diagnostic \\
4 & severe distortion & non-diagnostic \\
\hline
\end{tabular}

Table 1. Magnetic resonance imaging (MRI) protocol for the posterior cerebral fossa

\begin{tabular}{|c|c|c|c|c|c|}
\hline Sequence & $\begin{array}{l}\mathrm{TR} / \mathrm{TE} / \mathrm{TI} \\
\text { [ms] }\end{array}$ & $\begin{array}{c}\text { Slice thickness } \\
{[\mathrm{mm}]}\end{array}$ & $\begin{array}{l}\text { Inter-slice gap } \\
{[\mathrm{mm}]}\end{array}$ & $\begin{array}{c}\text { FoV } \\
{\left[\mathrm{mm}^{2}\right]}\end{array}$ & Matrix \\
\hline $\begin{array}{l}\text { Axial TSE } \\
\text { T1-weighted }\end{array}$ & $5,000 / 14$ & 5 & 1.5 & $240 \times 240$ & $256 \times 256$ \\
\hline $\begin{array}{l}\text { Axial TSE } \\
\text { T2-weighted }\end{array}$ & $4,000 / 102$ & 5 & 1.5 & $240 \times 240$ & $256 \times 256$ \\
\hline Axial FLAIR & $9,000 / 84 / 2,500$ & 5 & 1.5 & $240 \times 240$ & $256 \times 256$ \\
\hline
\end{tabular}

TR - repetition time; TE - echo time; TI - inversion time (for FLAIR); FoV - field of view; TSE - turbo spin-echo; T1 - longitudinal relaxation time; T2 - transverse relaxation time; FLAIR - fluid-attenuated inversion recovery. 

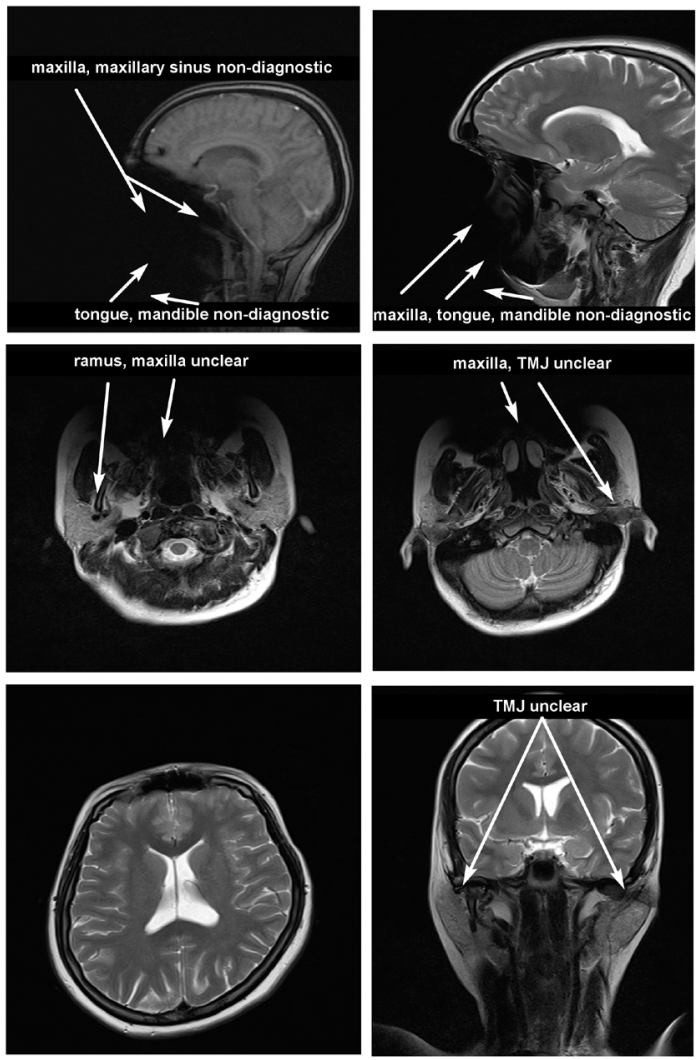

Fig. 1. Magnetic resonance imaging (MRI) scans of the head and neck region with $3 \mathrm{M}$ Unitek Gemini stainless steel buccal/labial brackets TMJ - temporomandibular joint.
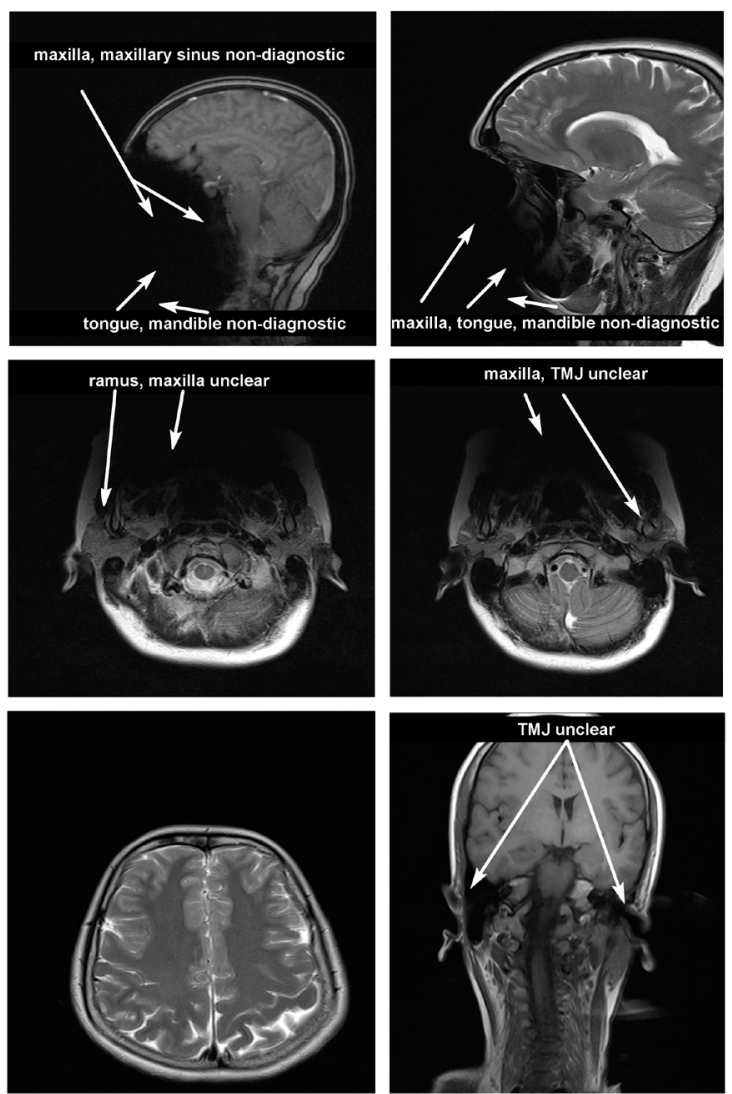

Fig. 2. Magnetic resonance imaging (MRI) scans of the head and neck region with Classic Orthodontics Invisible 7G stainless steel lingual brackets
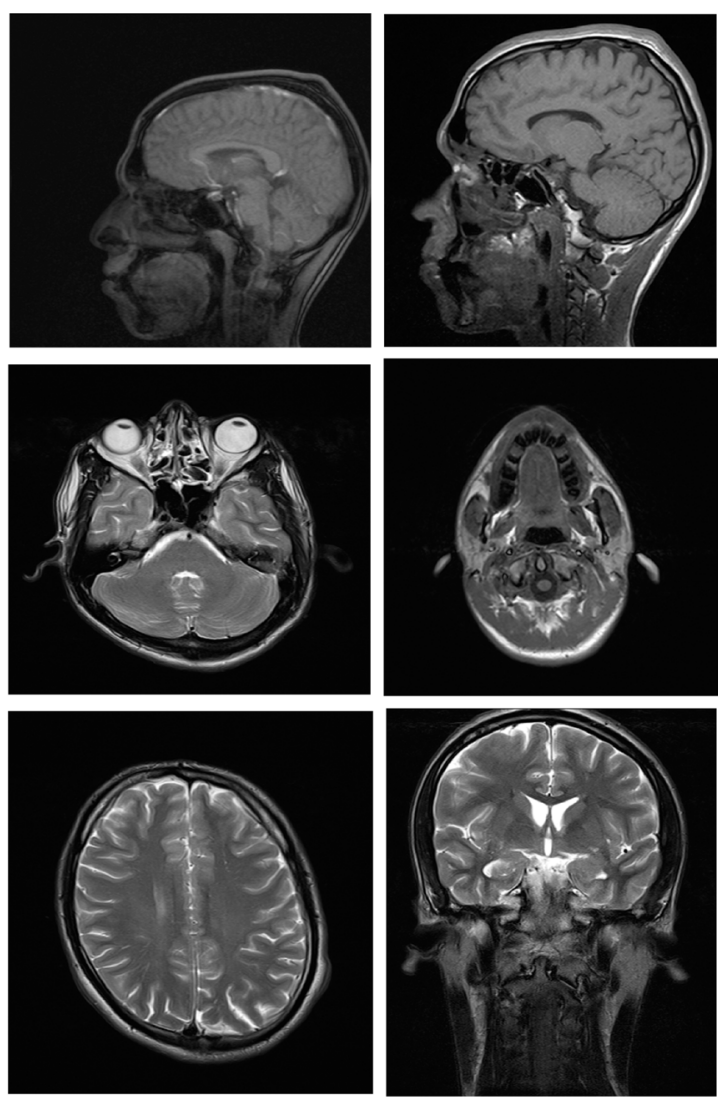

Fig. 3. Magnetic resonance imaging (MRI) scans of the head and neck region with 3M Unitek Clarity SL ceramic self-ligating brackets with metal slots
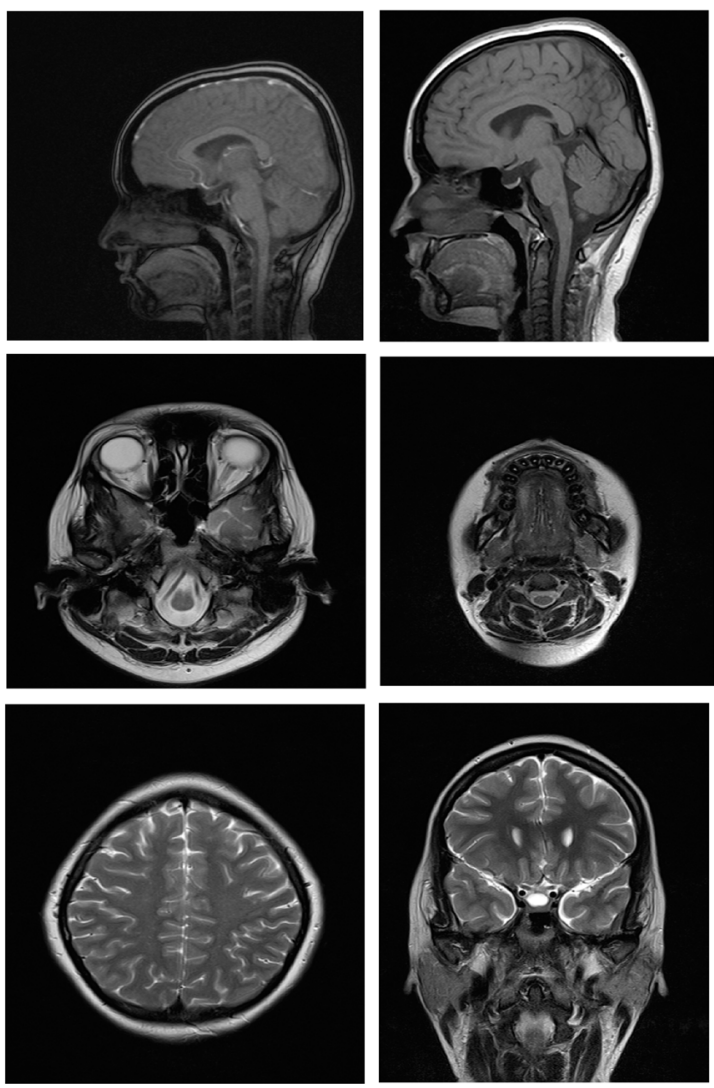

Fig. 4. Magnetic resonance imaging (MRI) scans of the head and neck region with Ormco Inspire ICE ceramic brackets 

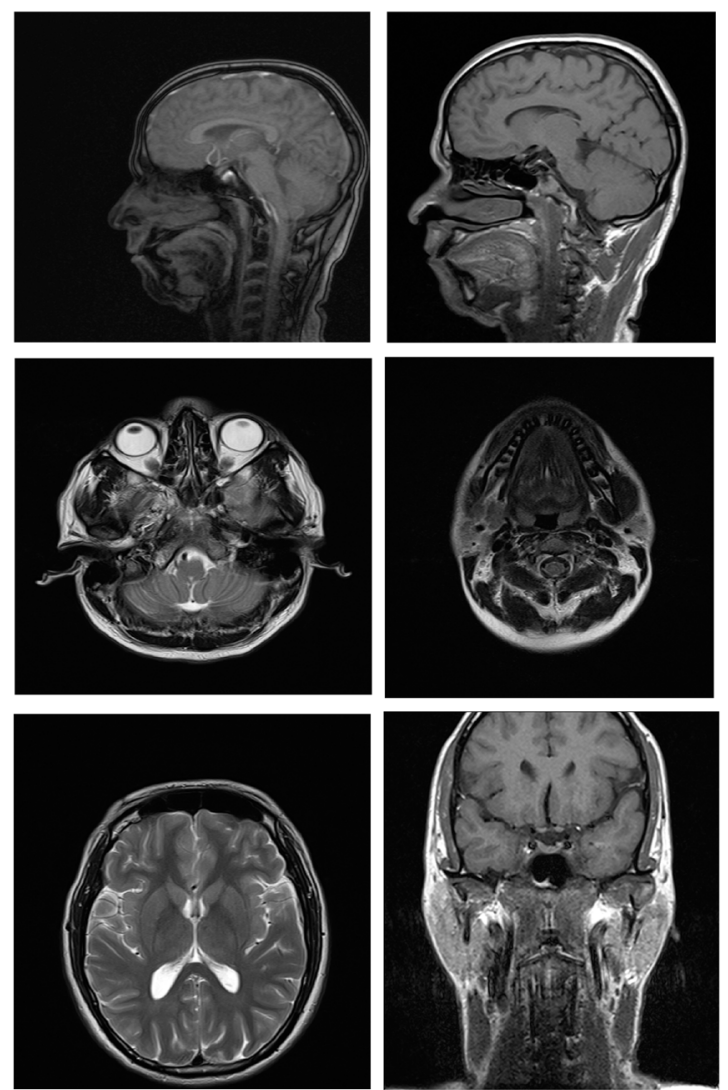

Fig. 5. Magnetic resonance imaging (MRI) scans of the head and neck region with JJ Orthodontics Ora-plus polycarbonate brackets
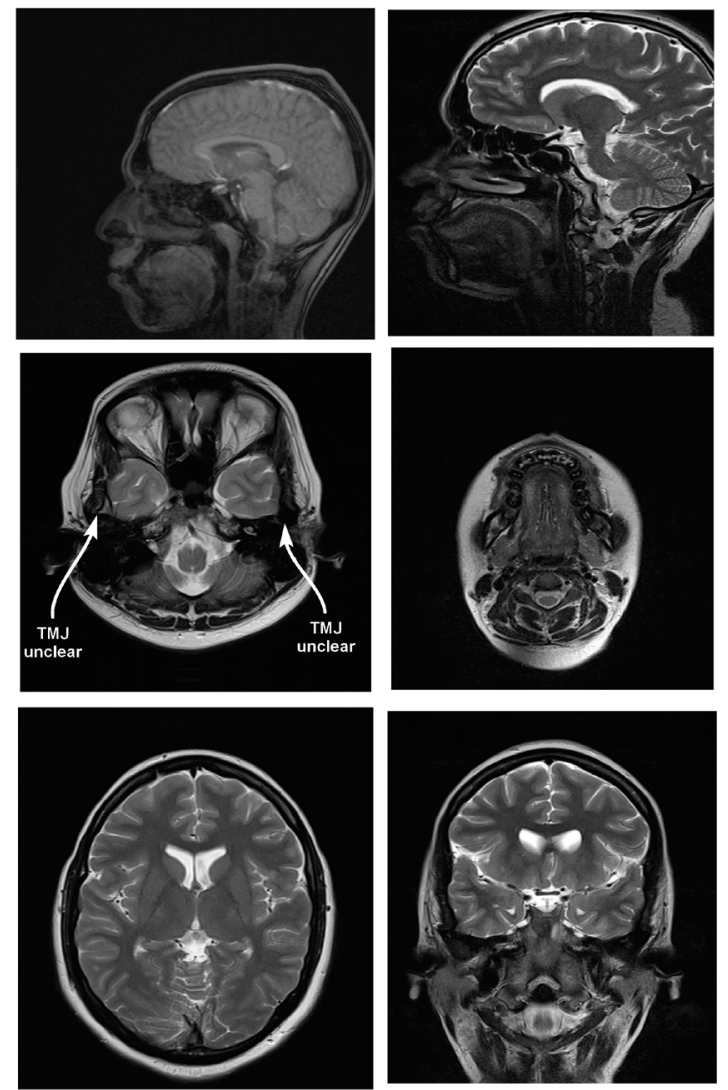

Fig. 6. Magnetic resonance imaging (MRI) scans of the head and neck region with an Ortho-Direct titanium retainer in the mandibular arch
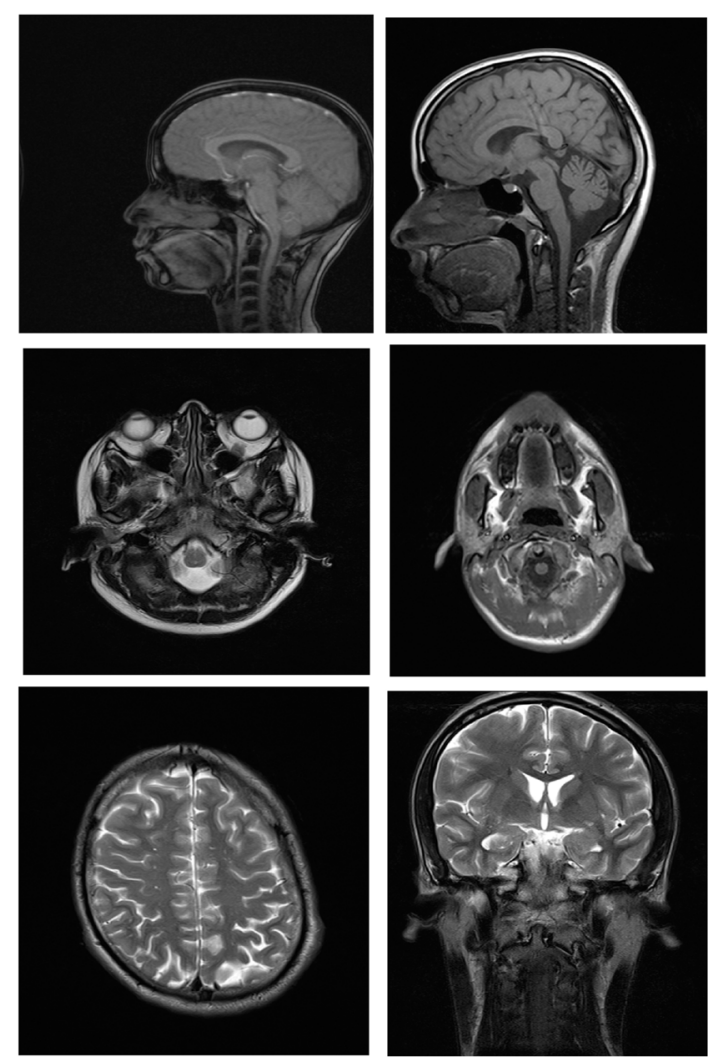

Fig. 7. Magnetic resonance imaging (MRI) scans of the head and neck region with a GC America everStick ORTHO fiber-reinforced composite retainer in the mandibular arch
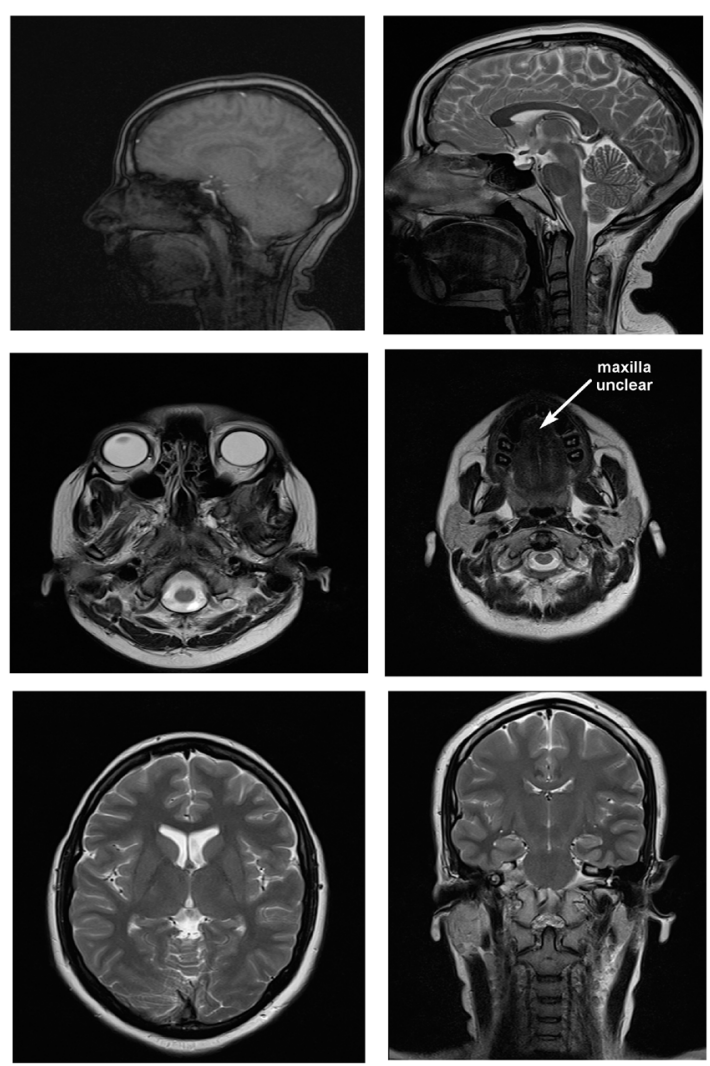

Fig. 8. Magnetic resonance imaging (MRI) scans of the head and neck region with multi-stranded stainless steel retainers in the maxillary and mandibular arches 

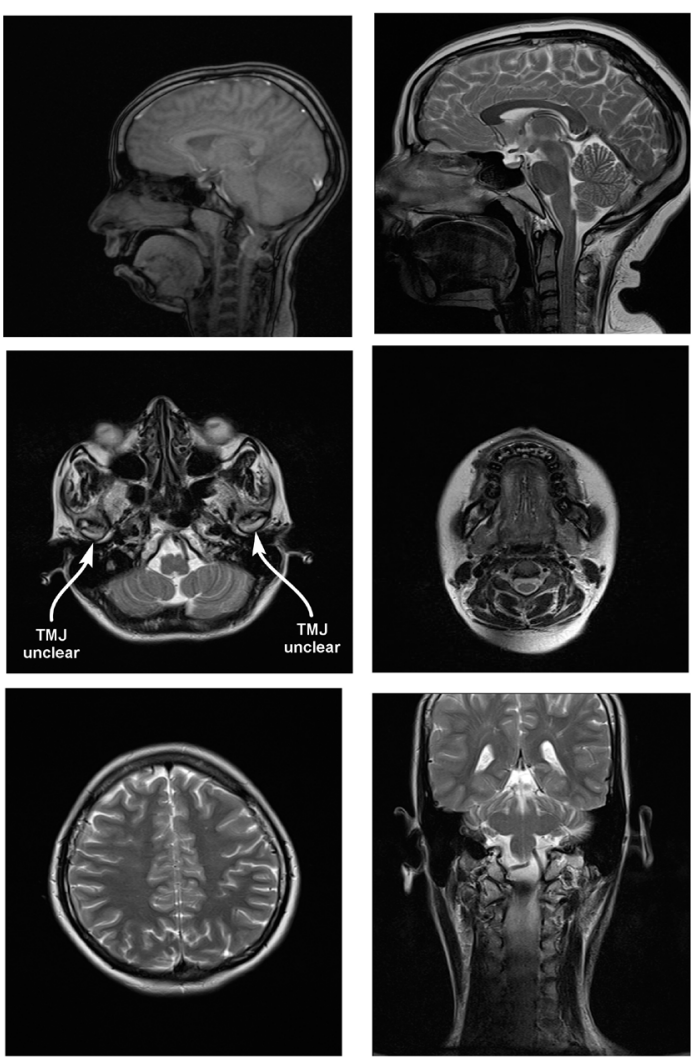

Fig. 9. Magnetic resonance imaging (MRI) scans of the head and neck region with a combination of a stainless steel retainer in the maxillary arch and a titanium retainer in the mandibular arch
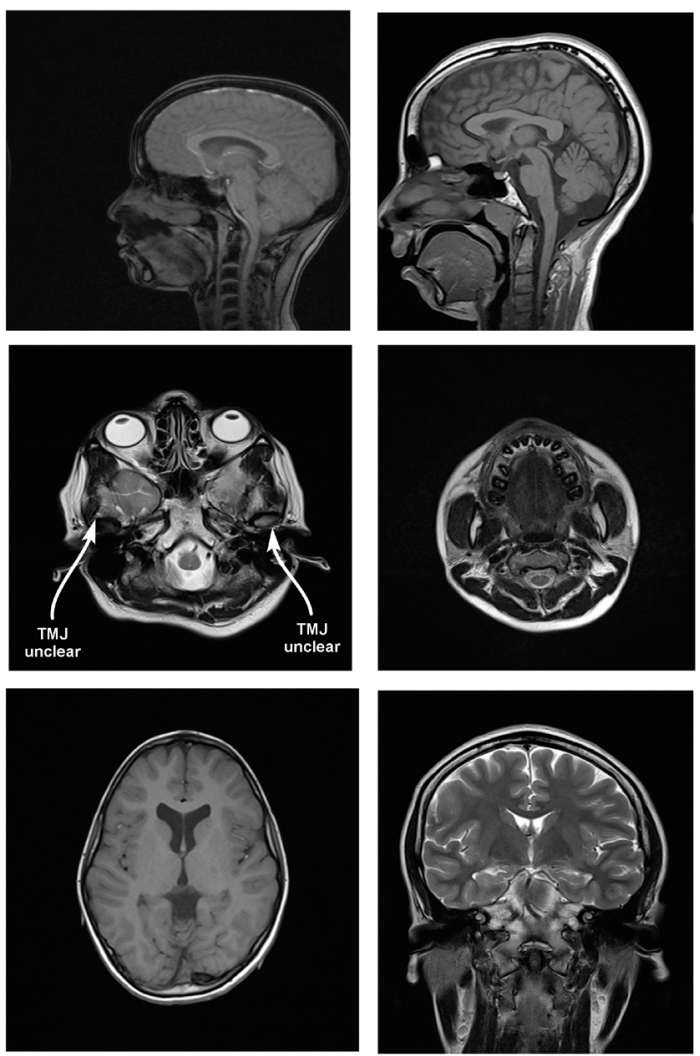

Fig. 10. Magnetic resonance imaging (MRI) scans of the head and neck region with a combination of a stainless steel retainer in the maxillary arch and a fiber-reinforced composite retainer in the mandibular arch

\section{Statistical analysis}

The statistical analysis was performed using the IBM SPSS Statistics for Windows software, v. 19.0 (IBM Corp., Armonk, USA). Non-parametric tests were used for the study. Differences between the materials and the anatomic sites with regard to the distortion rating scale were analyzed for statistical significance with the paired Wilcoxon signed-rank test. A $p$-value $<0.05$ was considered a statistically significant difference. The interrater agreement for each pair of evaluators was assessed for each material and each anatomic site with Cohen's kappa coefficient $(\kappa)$.

\section{Results}

Statistically significant differences were found between the mean distortion scores given to stainless steel brackets (both buccal/labial and lingual) and to other bracket and retainer materials as well as controls $(p<0.05)$ (Table 3$)$.

Table 3. Individual pairwise comparisons between various brackets and various retainers vs. stainless steel brackets (paired Wilcoxon signed-rank test)

\begin{tabular}{|c|c|c|}
\hline Comparison & Z-value & $p$-value \\
\hline $\begin{array}{l}\text { Ceramic SL brackets with metals slots } \\
\text { vs. SS brackets }\end{array}$ & -2.333 & $0.020^{*}$ \\
\hline $\begin{array}{l}\text { Ceramic brackets } \\
\text { vs. SS brackets }\end{array}$ & -2.264 & $0.024^{*}$ \\
\hline $\begin{array}{l}\text { Polycarbonate brackets } \\
\text { vs. SS brackets }\end{array}$ & -2.333 & $0.020^{*}$ \\
\hline $\begin{array}{l}\text { Ti retainer }(\mathrm{L}) \\
\text { vs. SS brackets }\end{array}$ & -2.333 & $0.020^{*}$ \\
\hline $\begin{array}{l}\text { FRC retainer }(L) \\
\text { vs. SS brackets }\end{array}$ & -2.264 & $0.024^{*}$ \\
\hline $\begin{array}{l}\text { SS retainers }(\mathrm{U} / \mathrm{L}) \\
\text { vs. SS brackets }\end{array}$ & -2.333 & $0.020^{*}$ \\
\hline $\begin{array}{l}\text { SS retainer }(U) / \text { Ti retainer }(\mathrm{L}) \\
\text { vs. SS brackets }\end{array}$ & -2.333 & $0.020^{*}$ \\
\hline $\begin{array}{l}\text { SS retainer }(U) / F R C \text { retainer }(L) \\
\text { vs. SS brackets }\end{array}$ & -2.333 & $0.020^{*}$ \\
\hline
\end{tabular}

SL - self-ligating; SS - stainless steel; Ti - titanium; FRC - fiber-reinforced composite; $U$ - upper arch; $L$ - lower arch; * statistically significant.

Table 4 presents the mean distortion scores for particular bracket and retainer materials based on the scores obtained for each subject. Each subject received a mean distortion score above 3 for stainless steel buccal and lingual brackets, indicating that the images were non-diagnostic. Each subject received a mean distortion score below 3 for other bracket and retainer materials, which indicates that these MR images were diagnostic. 
Table 4. Descriptive statistics regarding the distortion scores for all bracket and retainer materials

\begin{tabular}{|c|c|c|c|c|c|}
\hline Material & $N$ & $M$ & $S D$ & $\min$ & $\max$ \\
\hline SS buccal/labial brackets & 6 & 3.67 & 0.52 & 3 & 4 \\
\hline SS lingual brackets & 6 & 3.67 & 0.52 & 3 & 4 \\
\hline Ceramic SL brackets with metal slots & 6 & 1.67 & 0.52 & 1 & 2 \\
\hline Ceramic brackets & 6 & 1.00 & 0.00 & 1 & 1 \\
\hline Polycarbonate brackets & 6 & 1.00 & 0.00 & 1 & 1 \\
\hline Ti retainer $(L)$ & 6 & 2.00 & 0.00 & 2 & 2 \\
\hline FRC retainer $(\mathrm{L})$ & 6 & 1.00 & 0.00 & 1 & 1 \\
\hline SS retainers $(\mathrm{U} / \mathrm{L})$ & 6 & 2.00 & 0.00 & 2 & 2 \\
\hline SS retainer $(\mathrm{U})$ / Ti retainer $(\mathrm{L})$ & 6 & 2.00 & 0.00 & 2 & 2 \\
\hline SS retainer $(U) / F R C$ retainer $(U)$ & 6 & 2.00 & 0.00 & 2 & 2 \\
\hline
\end{tabular}

$N$ - number of evaluators; $M$ - mean; SD - standard deviation; min - minimum; max - maximum.

Table 5 details the mean distortion scores obtained for different types of brackets and retainers according to the anatomic sites for all subjects. All sequences and all radiologists were considered when determining these mean values. Except for stainless steel buccal/ labial and lingual brackets, other materials had an average distortion score below 3 with regard to various anatomic sites, indicating that these MR images were diagnostic. For stainless steel buccal/labial and lingual brackets, the mean distortion scores were above 3 consistently for each anatomic site except for the posterior cerebral fossa (a score of 1.16). This indicates that these MR images were non-diagnostic for all areas except for the posterior cerebral fossa.

Table 6 presents the results of the interrater reliability test with the use of Cohen's kappa coefficient $(\kappa)$, concerning the assessments of the 6 radiologists. Excellent consistency between the examiners was obtained with a $98 \%(0.986)$ statistical value.

Table 5. Descriptive statistics regarding the distortion scores for all bracket and retainer materials according to various anatomic sites

\begin{tabular}{|c|c|c|c|c|c|c|}
\hline Material & TMJ & Tongue & Maxilla & Mandible & Maxillary sinus & $\begin{array}{c}\text { Posterior } \\
\text { cerebral fossa }\end{array}$ \\
\hline SS buccal/labial brackets & $4.00 \pm 1.15$ & $4.00 \pm 1.15$ & $4.00 \pm 1.15$ & $4.00 \pm 1.15$ & $4.00 \pm 1.15$ & $1.16 \pm 0.86$ \\
\hline SS lingual brackets & $4.00 \pm 1.15$ & $4.00 \pm 1.15$ & $4.00 \pm 1.15$ & $4.00 \pm 1.15$ & $4.00 \pm 1.15$ & $1.16 \pm 0.86$ \\
\hline Ceramic SL brackets with metal slots & $1.60 \pm 0.24$ & $1.60 \pm 0.24$ & $1.60 \pm 0.24$ & $1.60 \pm 0.24$ & $1.60 \pm 0.24$ & $1.00 \pm 0.00$ \\
\hline Ceramic brackets & $1.00 \pm 0.00$ & $1.00 \pm 0.00$ & $1.00 \pm 0.00$ & $1.00 \pm 0.00$ & $1.00 \pm 0.00$ & $1.00 \pm 0.00$ \\
\hline Polycarbonate brackets & $1.00 \pm 0.00$ & $1.00 \pm 0.00$ & $1.00 \pm 0.00$ & $1.00 \pm 0.00$ & $1.00 \pm 0.00$ & $1.00 \pm 0.00$ \\
\hline Ti retainer $(\mathrm{L})$ & $2.16 \pm 0.42$ & $2.00 \pm 0.42$ & $2.00 \pm 0.42$ & $2.00 \pm 0.42$ & $2.00 \pm 0.42$ & $1.00 \pm 0.42$ \\
\hline FRC retainer $(L)$ & $1.00 \pm 0.00$ & $1.00 \pm 0.00$ & $1.00 \pm 0.00$ & $1.00 \pm 0.00$ & $1.00 \pm 0.00$ & $1.00 \pm 0.00$ \\
\hline SS retainers (U/L) & $2.16 \pm 0.42$ & $2.00 \pm 0.42$ & $2.00 \pm 0.42$ & $2.00 \pm 0.42$ & $2.00 \pm 0.42$ & $1.00 \pm 0.42$ \\
\hline SS retainer $(U) /$ Ti retainer $(\mathrm{L})$ & $2.16 \pm 0.65$ & $2.00 \pm 0.65$ & $2.00 \pm 0.65$ & $2.00 \pm 0.65$ & $2.00 \pm 0.65$ & $1.00 \pm 0.65$ \\
\hline SS retainer $(U) / F R C$ retainer $(L)$ & $2.00 \pm 0.45$ & $2.00 \pm 0.45$ & $2.00 \pm 0.45$ & $2.00 \pm 0.45$ & $2.00 \pm 0.45$ & $1.00 \pm 0.45$ \\
\hline
\end{tabular}

Data presented as $M \pm S D$

Table 6. Results of the interrater reliability test

\begin{tabular}{|c|c|c|c|c|c|c|}
\hline \multirow{2}{*}{$\begin{array}{l}\text { Interrater } \\
\text { reliability test }\end{array}$} & \multirow{2}{*}{$I C C$} & \multicolumn{2}{|c|}{$95 \% \mathrm{Cl}$} & \multicolumn{3}{|c|}{$F$ test with the true value 0} \\
\hline & & lower bound & upper bound & value & df1 & df2 \\
\hline Single measures & 0.922 & 0.829 & 0.977 & 81.250 & 9 & 45 \\
\hline Average measures & 0.986 & 0.967 & 0.996 & 81.250 & 9 & 45 \\
\hline
\end{tabular}

ICC - intraclass correlation coefficient with Cohen's kappa coefficient ( $\mathrm{K}$; $\mathrm{Cl}$ - confidence interval; $\mathrm{df}$ - degree of freedom. 


\section{Discussion}

The increasing availability of MRI machines and everimproving MRI technology has led to cranial MRI diagnostic techniques being used more frequently in medicine and other health-related fields. For example, over the past 10-15 years, MRI has improved our understanding and management of multiple sclerosis and other demyelinating disorders. ${ }^{18-20}$

With an increase in orthodontic awareness and growth in the number of orthodontists around the world, the number of patients seeking orthodontic treatment has also increased. Hence, it is expected that some patients undergoing orthodontic treatment or having fixed retainers after the completion of their orthodontic treatment may require MRI for various reasons. Radiologists currently ask orthodontists to remove orthodontic appliances before cranial MRI, as they lack the knowledge on orthodontic materials and their possible influence on the diagnostic image quality of MRI scans.

Previous studies have determined that some metals used in dentistry cause cranial MR image distortion. Lissac et al. discovered that many dental materials caused significant distortion of cranial MR images, and therefore should not be used for fixed dental work. ${ }^{21}$ Masumi et al. supported this observation by concluding that dental materials that cause MR image distortion adversely affected the diagnosis of abnormalities in the craniocervical region. ${ }^{22}$ Sadowsky et al. concluded that the areas closest to the problem material (metal) were the most distorted, while the anatomic sites farthest from the problem material (metal) were the least distorted. ${ }^{12}$

In the present study, stainless steel buccal/labial and lingual orthodontic brackets caused more distortion of cranial MR images and received higher distortion scores for all anatomic sites than other orthodontic bracket and retainer materials. The mean distortion score assigned by the radiologists to stainless steel appliances was 3.67. According to the anatomic landmarks, it was 4 by the same radiologists for TMJ, the tongue, the maxilla, the mandible, and the maxillary sinus, which means maximum distortion of the MR images. However, it was only 1.16 for the posterior cerebral fossa. This indicates that stainless steel buccal/labial and lingual brackets caused distortion that was most severe in the anatomic sites adjacent to them. The mean score of 1.16 for the posterior cerebral fossa means that there was almost no distortion, which shows a pattern of reduced artifacts with an increasing distance of the anatomic site from the brackets.

Since stainless steel buccal/labial and lingual brackets consistently had higher distortion scores, the MR images were considered non-diagnostic. The results of this study are similar to those obtained by Patel et al., ${ }^{8}$ Elison et al., ${ }^{17}$ Razdan and Rani, ${ }^{23}$ Beau et al., ${ }^{24}$ Zhylich et al., ${ }^{25}$ and Cassetta et $a .^{26}$ However, they are contradictory to the research done by Sadowsky et al., since an older version of an MRI machine was used in their study. ${ }^{12}$

Ceramic self-ligating brackets with metal slots had a mean distortion score of 1.67 and mean distortion scores of 1.6 at all 6 anatomic sites except for the posterior cerebral fossa (1.0). This indicates minimal distortion for all ROI. Hence, the MR images taken with self-ligating brackets were diagnostic. A study conducted by Asano et al., in which 3 types of brackets (titanium, ceramic and ceramic with metal slots) were used in a single patient, concluded that the MR images showed little distortion and were interpretable. ${ }^{27}$ Since all 3 types of brackets were used in a single patient, one cannot distinguish their individual effects on the MRI scans. ${ }^{27}$

The mean distortion scores for ceramic and polycarbonate brackets were 1.00, and the mean distortion scores at all 6 anatomic sites were also 1.00, which implies no distortion for any ROI; hence, the MR images with ceramic and polycarbonate brackets were considered diagnostic.

In recent years, many new fixed retainers have entered the market. Some of them include titanium retainers, fiber-reinforced composite retainers and braided titanium retainers. Retention is an important aspect in orthodontics. Many patients need to have fixed retainers for an extended period of time and may require MRI for various reasons during that time; hence, 3 types of fixed retainers and 2 combinations of them were evaluated for their influence on the diagnostic image quality of MRI scans. To date, there are no comprehensive MRI studies on these fixed retainers and combinations of these retainers in both arches.

Stainless steel retainers in both the maxillary and mandibular arch had a mean distortion score of 2.00. The mean distortion scores at the anatomic sites were 2.16 for TMJ, and 2.00 for the tongue, the maxilla, the mandible, and the maxillary sinus, with only the posterior cerebral fossa having a score of 1.00. This means that there was minimal or no distortion for all ROI. Hence, the MR images with stainless steel retainers in the maxillary and mandibular arches were considered diagnostic.

Titanium retainers in the mandibular arch alone had a mean distortion score of 2.00. The mean distortion scores at the anatomic sites were 2.16 for TMJ, 2.00 for the tongue, the maxilla, the mandible, and the maxillary sinus, and 1.00 for the posterior cerebral fossa, which indicates that there was minimal or no distortion for all ROI. Hence, the MR images with titanium retainers in the mandibular arch alone were considered diagnostic. The results of this study are similar to the findings reported in studies by Shalish et al., ${ }^{28}$ Beau et al. ${ }^{24}$ and Zhylich et al. ${ }^{25}$

Fiber-reinforced composite retainers in the mandibular arch alone had a mean distortion score of 1.00 and mean distortion scores of 1.00 at all 6 anatomic sites, which means there was no distortion for any ROI. Hence, the MR images with fiber-reinforced composite 
retainers in the mandibular arch only were considered diagnostic. There are no studies reporting on this type of retainers.

Sometimes, a combination of different fixed retainers is placed in the maxillary and mandibular arches based on the orthodontist's choice, the availability of retainers or the patient's preference. Hence, combinations of fixed retainers placed in the maxillary and mandibular arches were also examined in this study, as they have not been studied until now.

The combination of a stainless steel retainer in the maxillary arch and a fiber-reinforced retainer in the mandibular arch had a mean distortion score of 2.00 and mean distortion scores of 2.00 for TMJ, the tongue, the maxilla, the mandible, and the maxillary sinus; the posterior cerebral fossa had a score of 1.00. Therefore, the MR images with this combination caused minimal or no distortion, and were considered diagnostic by the radiologists.

The combination of a stainless steel retainer in the maxillary arch and a titanium retainer in the mandibular arch received similar scores to the previous combination. Therefore, the MR images with a stainless steel retainer in the maxillary arch and a titanium retainer in the mandibular arch were considered diagnostic.

\section{Conclusions}

Stainless steel buccal/labial and lingual brackets caused maximum distortion of the MR images, rendering them non-diagnostic; hence, they should be removed before MRI. Ceramic and polycarbonate brackets as well as fiber-reinforced composite retainers did not distort the images, so they need not be removed before MRI. Ceramic self-ligating brackets with metal slots, titanium retainers, stainless steel retainers, and combinations of these fixed retainers caused minimal distortion, but the MR images were still diagnostic. Hence, these types of materials may not need to be removed prior to MRI.

\section{ORCID iDs}

Praveen Kumar Neela (1) https://orcid.org/0000-0001-5168-4439 Venkat Kishan Tatikonda (1) https://orcid.org/0000-0002-8439-6473 Mohammed Wahajuddin Syed (10) https://orcid.org/0000-0003-4289-7402 Pavan Kumar Mamillapalli [1] https://orcid.org/0000-0003-2058-2864 Vasu Murthy Sesham (1) https://orcid.org/0000-0003-3238-8639 Sreekanth Keesara (1) https://orcid.org/0000-0001-6574-5327

\section{References}

1. Wu LM, Xu JR, Liu MJ, et al. Value of magnetic resonance imaging for nodal staging in patients with head and neck squamous cell carcinoma: A meta-analysis. Acad Radiol. 2012;19(3):331-340. doi:10.1016/j.acra.2011.10.027

2. Liedberg J, Panmekiate S, Petersson A, Rohlin M. Evidencebased evaluation of three imaging methods for the temporomandibular disc. Dentomaxillofac Radiol. 1996;25(5):234-241. doi:10.1259/dmfr.25.5.9161176
3. Kim DE, Bae HJ, Lee SH, Kim H, Yoon BW, Roh JK. Gradient echo magnetic resonance imaging in the prediction of hemorrhagic vs ischemic stroke: A need for the consideration of the extent of leukoariosis. Arch Neurol. 2002;59(3):425-429. doi:10.1001/archneur.59.3.425

4. Hauser WA. The prevalence and incidence of convulsive disorders in children. Epilepsia. 1994;35(Suppl 2):S1-S6. doi: 10.1111/j.15281157.1994.tb05932.x

5. Scheck SM, Boyd RN, Rose SE. New insights into the pathology of white matter tracts in cerebral palsy from diffusion magnetic resonance imaging: A systematic review. Dev Med Child Neurol. 2012;54(8):684-696. doi:10.1111/j.1469-8749.2012.04332.x

6. Mavreas D, Athanasiou AE. Factors affecting the duration of orthodontic treatment: A systematic review. Eur J Orthod. 2008;30(4):386-395. doi:10.1093/ejo/cjn018

7. Graber TM, Vanarsdall RL Jr., Vig KWL. Orthodontics: Current Principles and Techniques. $4^{\text {th }}$ ed. St. Louis, MO: Elsevier; 2005.

8. Patel A, Bhavra GS, O'Neill JRS. MRI scanning and orthodontics. J Orthod. 2006;33(4):246-249. doi:10.1179/146531205225021726

9. Harris TMJ, Faridrad MR, Dickson JAS. The benefits of aesthetic orthodontic brackets in patients requiring multiple MRI scanning. J Orthod. 2006;33(2):90-94. doi:10.1179/146531205225021465

10. Cacciafesta V. Difference in bonding/debonding chair time between a resin-modified, self-cured glass ionomer cement and a composite resin: A randomized controlled trial. Prog Orthod. 2001;2(1):9-13. doi:10.1034/j.1600-9975.2001.20103.x

11. Diedrich P. Enamel alterations from bracket bonding and debonding: A study with the scanning electron microscope. Am J Orthod. 1981;79(5):500-522. doi:10.1016/s0002-9416(81)90462-0

12. Sadowsky PL, Bernreuter W, Lakshminarayanan AV, Kenney P. Orthodontic appliances and magnetic resonance imaging of the brain and temporomandibular joint. Angle Orthod. 1988;58(1):9-20. doi:10.1043/0003-3219(1988)058<0009:OAAMRI>2.0.CO;2

13. Hinshaw DB Jr., Holshouser BA, Engstrom HI, Tjan AH, Christiansen EL, Catelli WF. Dental material artefacts on MR images. Radiology. 1988;166(3):777-779. doi:10.1148/radiology.166.3.3340777

14. Okano Y, Yamashiro M, Kaneda T, Kasai K. Magnetic resonance imaging diagnosis of the temporomandibular joint in patients with orthodontic appliances. Oral Surg Oral Med Oral Pathol Oral Radiol Endod. 2003;95(2):255-263. doi:10.1067/moe.2003.37

15. Różyło-Kalinowska I, Walawska B, Predko-Engel A, Jurkiewicz $E$, Urbanik A. Magnetic resonance imaging in orthodontic patients. Guidelines of the Polish Orthodontic Society (PTO), the Polish Medical Radiological Society (PLTR), and the Polish Dental Association (PTS). J Stoma. 2019;72(1):1-3. doi:10.5114/jos.2019.86484

16. Regier M, Kemper J, Kaul MG, et al. Radiofrequency-induced heating near fixed orthodontic appliances in high field MRI systems at 3.0 Tesla. J Orofac Orthop. 2009;70(6):485-494. doi:10.1007/s00056-009-9923-0

17. Elison JM, Leggitt VL, Thomson M, Oyoyo U, Wycliffe ND. Influence of common orthodontic appliances on the diagnostic quality of cranial magnetic resonance images. Am J Orthod Dentofacial Orthop. 2008;134(4):563-572. doi:10.1016/j.ajodo.2006.10.038

18. Fleiss JL. Statistical Methods for Rates and Proportions. $2^{\text {nd }}$ ed. New York, NY: John Wiley \& Sons; 1981:128-136.

19. Miller DH, Grossman RI, Reingold SC, McFarland HF. The role of magnetic resonance techniques in understanding and managing multiple sclerosis. Brain. 1998;121(Pt 1):3-24. doi:10.1093/brain/121.1.3

20. Engelbrecht V, Scherer A, Rassek M, Witsack HJ, Mödder U. Diffusion-weighted MR imaging in the brain in children: Findings in the normal brain and in the brain with white matter diseases. Radiology. 2002;222(2):410-418. doi:10.1148/radiol.2222010492

21. Lissac M, Metrop D, Brugirard J, et al. Dental materials and magnetic resonance imaging. Invest Radiol. 1991;26(1):40-45. doi:10.1097/00004424-199101000-00008.

22. Masumi S, Arita M, Morikawa M, Toyoda S. Effect of dental metals on magnetic resonance imaging (MRI). J Oral Rehabil. 1993;20(1):97-106. doi:10.1111/j.1365-2842.1993.tb01519.x

23. Razdan D, Rani MS. Magnetic resonance imaging artifacts caused by brackets of various materials - an in vivo study. APOS Trends Orthod. 2011;2(1):1-5.

24. Beau A, Bossard D, Gebeile-Chauty S. Magnetic resonance imaging artefacts and fixed orthodontic attachments. Eur J Orthod. 2015;37(1):105-110. doi:10.1093/ejo/cju020 
25. Zhylich D, Krishnan $P$, Muthusami $P$, et al. Effects of orthodontic appliances on the diagnostic quality of magnetic resonance images of the head. Am J Orthod Dentofacial Orthop. 2017;151(3):484-499. doi:10.1016/j.ajodo.2016.07.020

26. Cassetta M, Pranno N, Stasolla A, et al. The effects of a common stainless steel orthodontic bracket on the diagnostic quality of cranial and cervical 3T-MR images: A prospective, case-control study. Dentomaxillofac Radiol. 2017;46(6):20170051. doi:10.1259/dmfr.20170051

27. Asano S, Fukuda T, Saito K, Negishi S, Kasai K, Kaneda T. Metal artifacts caused by orthodontic self-ligation brackets on 1.5 Tesla MRI: Phantom study of various sequence including diffusion weighted imaging. Dent Radiol. 2015;55(1/4):86-91. doi:10.11242/dentalradiology.55.86

28. Shalish M, Dykstein N, Friedlander-Barenboim S, Ben-David E, Gomori JM, Chaushu S. Influence of common fixed retainers on the diagnostic quality of cranial magnetic resonance images. Am J Orthod Dentofacial Orthop. 2015;147(5):604-609. doi:10.1016/j.ajodo.2014.11.022. 